\title{
ATENÇÃO PRIMÁRIA À SAÚDE E APOIO PSICOSSOCIAL A CRIANÇAS E ADOLESCENTES VIITIMAS DE VIOLÊNCIA DOMÉSTICA: REVISÃO INTEGRATIVA
}

\section{PRIMARY HEALTHCARE AND PSYCHOSOCIAL SUPPORT TO CHILDREN AND ADOLESCENTS VICTIMIZED BY DOMESTIC VIOLENCE: AN INTEGRATIVE REVIEW}

Vanessa Julinda Ribeiro Coutinho Marques', Ana Beatriz Ribeiro Cavalcante Silval, Aryana Marques da Nóbrega Ayres', Camilla Rolim Pagels', Leonardo Pereira Tonil ${ }^{\prime}$, Cleyton Cezar Souto Silvalli*

\begin{abstract}
Resumo. A violência doméstica contra crianças e adolescentes é uma prática recorrente no Brasil e fere os direitos desse grupo, estabelecidos pela legislação vigente. Tais vítimas necessitam de apoio psicossocial, durante o acompanhamento pelo sistema de saúde. O trabalho tem como objetivo avaliar a oferta de apoio psicossocial para crianças e adolescentes, vítimas de violência doméstica, no contexto da Atenção Primária à Saúde. Trata-se de uma revisão de literatura, realizada pela análise de estudos científicos selecionados na Biblioteca Virtual de Saúde (BVS), através das bases de dados Scientific Eletronic Library Online (SciELO), Literatura Latino-Americana e do Caribe em Ciências da Saúde (LILACS) e PubMed, utilizando os Descritores em Ciências da Saúde (DeCS): "Serviços de Saúde Mental”, "Defesa da Criança e do Adolescente", "Violência”. Observou-se que a rede de apoio, apesar de possuir um aparato legal que ofereça proteção aos direitos das crianças e adolescentes, vítimas de violência, encontra-se desarticulada entre seus setores, o que dificulta o apoio psicossocial às vítimas. Conclui-se que são necessárias ações intersetoriais que visem integrar os elementos da rede de apoio, para que se possa oferecer um cuidado integral aos jovens vítimas de maus-tratos.
\end{abstract}

PALAVRAS-CHAVE: Serviços de Saúde Mental. Defesa da Criança e do Adolescente. Violência.

Abstract. Domestic violence against children and adolescents is a recurrent practice in Brazil, breaching the rights of this group as safeguarded by the current legislation. These victims need psychosocial assistance in the healthcare system. The aim of this paper is to assess the provision of psychosocial support to children and adolescents victims of violence, at the Primary Health Care. This is a literature review, conducted by analyzing scientific studies selected in the Virtual Health Library $(\mathrm{VHL})$, using the Scientific Electronic Electronic Library Online (SciELO), the Latin American and Caribbean Literature in Health Sciences (LILACS) and PubMed as databases. Also, we used the Health Sciences Descriptors (DeCS): "Mental Health Services", "Child Welfare", "Violence". It was observed that, in spite of having a legal provision safeguarding the rights of children and adolescents victims of violence, the support network is not well articulated among its sectors, hampering the access of these victims to psychosocial support. Therefore, intersectoral actions are necessary in order to integrate the support network elements, so that young victims of maltreatment have access to comprehensive care.

KEYWORDS: Mental Health Services. Child Welfare. Violence.

'Graduando (a). Curso de Medicina da Faculdade de Medicina Nova Esperança. Departamento de Medicina - FAMENE. CEP: 58067-695, João Pessoa, Parába, Brasil. ORCID ID: 0000-0002-4250-2223; 0000-0002-7522-6798; 0000-0002-5024-8173; 0000-0002-2045-1330.

"Graduando. Curso de Medicina do Centro Universitário de João Pessoa - UNIPÊ. Departamento de Medicina. CEP: 58053-000, João Pessoa, Paraíba, Brasil. ORCID ID: 0000-0002-8624-8706

III Doutor em Enfermagem na Atenção à Saúde. Programa de Pós Graduação em Saúde da Família da Faculdade de Enfermagem e Medicina Nova Esperança - FACENE. CEP: 58067-695, João Pessoa, Paraíba, Brasil. *Autor correspondente: cleyton.silva@famene.com.br. ORCID ID: 0000-0002-6187-0187. 


\section{INTRODUÇÃO}

A violência intrafamiliar contra crianças e adolescentes é definida como atos ou omissões praticados por pais, parentes ou responsáveis, podendo ser de natureza psicológica, física e/ou sexual, com o potencial de causar dor e sofrimento aos indivíduos acometidos. ${ }^{1}$ Essa definição incorpora dois elementos centrais: a evidência de comportamento prejudicial à criança e a presença de danos decorrentes dessa conduta. Ainda abrange omissão, supressão e transgressão dos direitos da criança e do adolescente. $^{2}$ Entre os efeitos resultantes dessa prática, observam-se comportamentos não adaptativos, déficits emocionais e até transtornos mentais graves, tais como: comportamento impulsivo, transtorno de hiperatividade, problemas de aprendizado escolar, transtornos de conduta e abuso de substâncias psicoativas. ${ }^{1,2}$

Um relatório da organização não governamental (ONG) World Vision estima que até 85 milhões de crianças e adolescentes, entre 2 e 17 anos, poderão se somar às vítimas de violência física, emocional e sexual em todo o planeta. O número representa um aumento que pode variar de 20 a 32\% da média anual das estatísticas oficiais. O confinamento em casa, essencial para conter a pandemia do novo coronavírus, acaba expondo essa população a uma maior incidência de violência doméstica, segundo dados da ONG. ${ }^{3}$

A Sociedade Brasileira de Pediatria (SBP) firmou parceria com o Conselho Federal de Medicina (CFM) e o Ministério dos Direitos Humanos, para buscar soluções contra agressões às crianças e adolescentes. Assim, como frutos dessa parceria, em 2011, foi lançado pela Sociedade de Pediatria de São Paulo (SPSP), com o apoio do CFM, o primeiro Manual de atendimento às crianças e adolescentes vítimas de violência, sendo a segunda edição do manual disponibilizada em 2018. Diariamente, são notificadas no Brasil, em média, 233 agressões de diferentes tipos (física, psicológica e tortura) contra crianças e adolescentes com idade até 19 anos. 4

Do total de casos notificados pelos serviços de saúde, 69,5\% (59.293) são decorrentes de violência física; $27,1 \%$ (23.110) de violência psicológica; e 3,3\% (2.890) de episódios de tortura. ${ }^{4}$ Não foram consideradas variações como violência e assédio sexual, abandono, negligência, trabalho infantil, entre outros tipos de agressão, que foram abordadas em uma nota de alerta publicada pela SBP. 4,5

Pelos dados do Sistema de Informação de Agravos de Notificação (SINAN) ${ }^{4}$, as populações pediátricas em situação de maior risco à violência são os faixas de 10 a 14 anos (20.773 ocorrências) e de 15 a 19 anos (44.203 notificações) em 2017. Juntas, contabilizando 66.976 casos. Em 2009, esses dois segmentos somaram 9.309 e entre 2009 e 2017 , o volume de notificações em jovens de 10 a 19 anos aumentou sete vezes.

No Brasil, a violência contra esse grupo adquiriu proporções alarmantes, tornando-se um grave problema de saúde pública. Foi ao final do ano de 1980 , que passou a ter maior atenção das autoridades competentes, com a promulgação da Constituição Federal de 1988 e com a Lei n. 8.069, do Estatuto da Criança e do Adolescente (ECA), promulgada em $1990 .^{2}$ De acordo com o Art. 87 do ECA, são linhas de ação da política de atendimento: políticas sociais básicas; programas e benefícios de assistência social; serviços especiais de prevenção, atendimento médico e psicossocial às vítimas de violência; 
proteção jurídico-social; políticas e programas destinados a prevenir ou abreviar o período de afastamento do convívio familiar; campanhas de estímulo ao acolhimento aos afastados do convívio familiar e à adoção. 5

Assim, surge 0 atendimento psicossocial, como estratégia no atendimento de crianças e adolescentes vítimas de violência, tornando imprescindível que os serviços oferecidos se articulem com a rede de atenção em volta da família. A intervenção psicossocial refere-se a uma prática capaz de empoderar o sujeito e incentivar a busca de soluções para suas dificuldades, em consonância com o contexto biopsicossocial em que o sujeito está inserido. ${ }^{6}$ Para que possa ocorrer, necessita de uma rede articulada, de modo a envolver diversos órgãos capazes de estabelecer ações terapêuticas, legais e de proteção, tais como: Centros de Referência da Assistência Social (CRAS), Centros de Referência Especializados da Assistência Social (CREAS), Conselhos de Direitos, Conselhos Tutelares, Juizado da Vara da Infância e Juventude, além de instituições que prestam atendimento, como escolas e unidades de saúde. ${ }^{1,6}$

No contexto da saúde, após o processo de Reforma Psiquiátrica no país, instituiu-se a Rede de Atenção Psicossocial (RAPS), responsável pelos cuidados em saúde

\section{MATERIAL E MÉTODOS}

Trata-se de uma revisão de literatura realizada através de estudos científicos que abordam o apoio psicossocial para crianças e adolescentes vítimas de violência, como preconizado pelo ECA.

Os artigos foram selecionados na Biblioteca Virtual de Saúde (BVS), através dos bancos de dados Scientific Electronic Library mental para os cidadãos. O Centro de Atenção Psicossocial Infantil (CAPSi) é o dispositivo ordenador da RAPS em demandas infantojuvenis, por meio de serviços especializados para esse grupo. Todavia, o CAPSi não abrange grande parte dos municípios brasileiros, fato que transfere a responsabilidade, nesses locais, para a Estratégia de Saúde da Família (ESF). ${ }^{7}$

Apesar das legislações e aparatos que garantam a proteção e os direitos das crianças e adolescentes, ainda existem dados mínimos de notificação pelos órgãos competentes em relação a essa problemática, evidenciando a despreocupação e o despreparo, tanto dos profissionais quanto da sociedade civil, em garantir os direitos infanto-juvenis. ${ }^{6}$

Faz-se necessário, assim, fortalecer a discussão do tema proposto devido à importância do combate à violência vivenciada por crianças e adolescentes. Além disso, é preciso entender os pontos falhos do apoio psicossocial prestado pela Atenção Primária à Saúde a essas vítimas relacionado ao aparato legal existente para garantir os direitos desse grupo.

Desse modo, o objetivo do trabalho é apresentar uma revisão na literatura acerca do apoio psicossocial para crianças e adolescentes vítimas de violência no contexto da Atenção Primária à Saúde.

Online (SciELO), Literatura Latino-Americana e do Caribe em Ciências da Saúde (LILACS) e PubMed.

Para tanto, empregaram-se os seguintes Descritores em Ciências da Saúde (DeCS): Serviços de Saúde Mental, Defesa da Criança e do Adolescente, Violência. Para as pesquisas na biblioteca eletrônica PubMed 
utilizaram-se Medical Subject Terms (MeSHTerms): Child Abuse, Mental Health e Adolescent.

Para inclusão na revisão, foram usados os seguintes critérios: 1) artigos disponíveis na íntegra, no idioma português e/ou inglês, publicados no período de 2012 a 2020; 2) temáticas envolvendo problemas psicossociais com crianças e adolescentes; 3) correlação com a Atenção Primária à Saúde. Além disso, a Lei n. 8.069, de 13 de julho de 1990, foi utilizada como comparativa entre o ideal a ser preconizado para esses casos e a realidade existente em diversas regióes do

\section{RESULTADOS}

Após a busca nas bases, foram encontrados um total de 16 produções no SciELO, sendo que apenas 6 se encaixaram nos critérios de inclusão. São eles: 3 periódicos e 3 artigos. Também 26 produções no LILACS, sendo apenas 3 artigos incluídos e selecionados conforme os critérios; e 5 produções no PubMed, mas apenas 1 artigo se encaixou nos critérios. Dessa forma, as 10 produções foram selecionadas pelos critérios de inclusão, organizados, lidos na íntegra e categorizados para posterior análise dos temas relevantes.

Nesse sentido, os temas comuns encontrados, após análise comparativa entre o apoio psicossocial às crianças e adolescentes pela Atenção Primária à Saúde discutidos nos resultados foram: assistência integral e apoio psicossocial da Atenção Primária à Saúde às vítimas de violência infanto-juvenil; a corresponsabilidade da família nos casos de violência doméstica; e, consequência dos maus-tratos a
Brasil.

Foram excluídos os artigos que abordavam a temática de violência sexual, por se tratar de um tema mais específico e com particularidades a serem debatidas em uma revisão específica. Também foram excluídas dissertações e teses. Outro critério de exclusão foi a ausência de relação com a Atenção Primária à Saúde, com exceção de um artigo selecionado no PubMed, sendo incluído pela relevância do tema abordado e trazer um panorama geral das consequências, a longo prazo, do abuso físico, do abuso emocional e da negligência infantil.

crianças e adolescentes.

\section{Artigos analisados}

Os artigos selecionados, a partir do cruzamento dos descritores "Serviços de Saúde Mental”, "Defesa da Criança e do Adolescente", "Violência" abordaram a articulação entre a Atenção Primária à Saúde e os demais órgãos competentes CRAS, CREAS, Conselhos de Direitos, Conselhos Tutelares, Juizado da Vara da Infância e Juventude, bem como o apoio psicossocial oferecido às crianças e adolescentes vítimas de violência doméstica.

Entre 2012 e 2020 foram publicados 47 artigos nas bases de dados pesquisadas, sendo 16 publicados na SciELO, 26 na LILACS e 5 no PubMed. Dentre esses, foram selecionadas criteriosamente 10 produções, das quais foram utilizados artigos de revisão narrativa e sistemática para a realização do estudo (Tabela 1). 
TABELA 1: Síntese dos artigos incluídos na revisão integrativa

\begin{tabular}{|c|c|c|c|}
\hline Título do trabalho & Autores & Ano & Base de Dados \\
\hline $\begin{array}{l}\text { Atendimento psicossocial a crianças e adolescentes em situação de } \\
\text { violência: o psicólogo e a rede de atenção }\end{array}$ & Macedo \& Conceição 6 & 2017 & SciELO \\
\hline $\begin{array}{l}\text { Cuidado em saúde mental infantil no contexto da Estratégia da } \\
\text { Saúde da Família: estudo avaliativo }\end{array}$ & Carvalho, Duarte, Glanzner ${ }^{7}$ & 2020 & SCIELO \\
\hline $\begin{array}{l}\text { Maus-tratos na infância e adolescência: percepção e conduta de } \\
\text { profissionais de nível superior que atuam na Estratégia Saúde da } \\
\text { Família }\end{array}$ & $\begin{array}{l}\text { Condori, Nascimento, Mitie, } \\
\text { Pizzatto, Mazza, Buffon }\end{array}$ & 2018 & SCIELO \\
\hline Violência doméstica contra crianças e adolescentes: um desafio & $\begin{array}{l}\text { Zambon, Jacintho, Medeiros, } \\
\text { Guglielminetti, Marmo }\end{array}$ & 2012 & SCiELO \\
\hline $\begin{array}{l}\text { Práticas profissionais que silenciam a violência intrafamiliar contra } \\
\text { crianças e adolescentes }\end{array}$ & $\begin{array}{l}\text { Schek, Silva, Lacharité, Cézar- } \\
\text { Vaz, Bueno, Ventura9 }\end{array}$ & 2018 & LILACS \\
\hline $\begin{array}{l}\text { Rede de proteção na assistência às crianças, adolescentes e suas } \\
\text { famílias em situação de violência }\end{array}$ & $\begin{array}{l}\text { Melo, Carlos, Freitas, Roque, } \\
\text { Aragão, Ferriani }^{1}\end{array}$ & 2020 & LILACS \\
\hline $\begin{array}{l}\text { A responsabilidade dos profissionais de saúde na notificação dos } \\
\text { casos de violência contra crianças e adolescentes de acordo com } \\
\text { seus códigos de ética }\end{array}$ & $\begin{array}{l}\text { Almeida, } \\
\text { Marques }^{10}\end{array}$ & 2012 & LILACS \\
\hline $\begin{array}{l}\text { Violência doméstica e risco para problemas de saúde mental em } \\
\text { crianças e adolescentes }\end{array}$ & $\begin{array}{l}\text { Hildebrand, Celeri, Morcillo, } \\
\text { Zanolli }^{11}\end{array}$ & 2015 & SCiELO \\
\hline $\begin{array}{l}\text { Atenção básica e cuidado colaborativo na atenção psicossocial de } \\
\text { crianças e adolescentes: facilitadores e barreiras }\end{array}$ & Teixeira, Couto, Delgado ${ }^{12}$ & 2017 & SCiELO \\
\hline $\begin{array}{l}\text { Violência doméstica contra crianças e adolescentes: um desafio The } \\
\text { long-term health consequences of child physical abuse, emotional } \\
\text { abuse, and neglect: a systematic review and meta-analysis }\end{array}$ & $\begin{array}{l}\text { Norman, Byambaa, Rumna, } \\
\text { Butchart, Scott, Vos } 13\end{array}$ & 2012 & PubMed \\
\hline
\end{tabular}

\section{DISCUSSÃO}

\section{Assistência integral e apoio psicossocial da Atenção Primária à Saúde às vítimas de violência infanto-juvenil}

Em relação à Atenção Primária à Saúde, materializada na ESF, o acolhimento torna-se de extrema importância durante o atendimento de crianças e adolescentes vítimas de violência doméstica, visto que é a porta de entrada para que se possa criar um vínculo com a vítima e com sua família. Por conseguinte, a escuta deve ser empática, acolhedora e isenta de julgamentos morais, de modo a possibilitar a integralidade e, também, a longitudinalidade do cuidado com a família. ${ }^{6}$ A Visita Domiciliar (VD) também é essencial como recurso de identificação de possíveis vítimas, pois possibilita ao Agente Comunitário de Saúde (ACS) a imersão no contexto domiciliar das famílias da localidade. 7
Em estudo realizado em Porto Alegre, verificou-se que uma ESF apresentou dificuldades no cuidado à saúde mental infantil durante o processo avaliativo, devido à insatisfatória articulação com o Conselho Tutelar e com o CRAS. Sendo assim, evidenciase a necessidade de intensificar as reuniões de equipe para elaboração de planos de cuidado, de modo a aperfeiçoar o trabalho intersetorial e, também, a otimizar a articulação com os demais órgãos competentes.7

Outro estudo ${ }^{8}$ relacionou os baixos índices de notificações das violências por parte dos profissionais à falta de informação durante a graduação, uma vez que, apesar do acesso a informações sobre maus-tratos contra crianças e adolescentes, não obtiveram treinamento, o que os torna incapazes de identificar os casos suspeitos. Além disso, também surgem outros fatores que impedem a notificação, 
como a ideia do sigilo profissional, medo das obrigações legais decorrentes e ineficiência dos órgãos oferecidos pelo ECA (Conselho Tutelar, Ministério Público, Defensoria Pública, Justiça da Infância e Juventude e Delegacias Especializadas). A baixa quantidade de notificações dificulta a quantificação dos casos e, assim, o conhecimento da real dimensão do problema. $^{2}$

De acordo com estudo de Schek et al.9, a falta de comunicação entre os profissionais que atuam em serviços de referência e contrarreferência também é definitiva na perpetuação da problemática, já que a desarticulação entre os profissionais envolvidos no tratamento pode resultar em casos arquivados e considerados resolvidos, mesmo sem estarem, de modo a silenciar a violência sofrida pelas vítimas. Ademais, também foi verificado que grande parte dos profissionais, ao encaminharem as vítimas para outros setores, não as orientam sobre como alcançar o setor encaminhado, transferindo totalmente a responsabilidade para o profissional de referência.

Verificou-se também que não há tanta preocupação, por parte dos profissionais, em atender os agressores e intervir na situação, pois consideram que não é algo de suas atribuições. Aqueles que atuam em hospitais, Unidades Básicas de Saúde e Conselhos Tutelares devem ter em mente que as vítimas retornarão para seus lares e estarão sujeitas a novas agressões, portanto suas ações não podem ser localizadas nem sequer momentâneas. ${ }^{9}$

Em uma entrevista realizada com profissionais do CREAS de Pernambuco, apesar de demonstrarem entendimento sobre a importância da RAPS, eles reconheceram que o serviço onde atuam ainda não consegue efetuar a proteção a esse público, o que está relacionado à falta de estrutura e à descontinuidade do atendimento prestado pelo serviço de assistência social. Também relataram que a atuação é limitada em muitos momentos, devido à ausência de uma normatização que direcione e padronize a assistência, à inexistência de um fluxo adequado das demandas e à inconstância nos atendimentos por parte do serviço. ${ }^{1}$

\section{A corresponsabilidade da família nos casos de violência doméstica}

A dinâmica familiar também representa um empecilho à detecção de casos suspeitos, pois a ideia de que a violência faz parte do processo de educação ainda faz parte da mentalidade de grande parte dos pais brasileiros até os dias atuais. $8,9,10 \mathrm{~A}$ perpetuação da violência doméstica contra crianças e adolescentes tem sido considerada instrumento de socialização e, logo, mecanismo automático a desobediências e a rebeldias. A manifestação desse padrão de comportamento parental pode ser percebida na taxa de mortalidade infantil brasileira, uma das mais elevadas na América Latina, ainda que tenha diminuído nos últimos anos. Desse modo, é dever do setor saúde atender os efeitos da violência nos jovens, seja na Atenção Primária à Saúde, atuando, principalmente, na prevenção e no monitoramento, seja na Atenção Especializada, por meio da reparação de traumas e lesões físicas nos serviços de urgência e emergência. ${ }^{10}$

Hildebrand et al. ${ }^{11}$ relataram em estudo que $96,8 \%$ dos responsáveis pelas agressões direcionadas a crianças e adolescentes também foram vítimas de violência na infância, evidenciando o padrão cíclico e cultural dessa prática. Ainda, obteveram que os principais agressores eram apenas a mãe (42,9\%), pai e mãe $(33,3 \%)$. Com menor frequência, apenas o pai apareceu em 13,9\% dos casos, padrasto/ madrasta/tio/tia em 4,4\% dos casos, avô/avó em $2 \%$. Ainda segundo a pesquisa, os sintomas mais comumente encontrados em infantes e jovens vítimas de violência 
doméstica foram problemas de conduta $(66,3 \%)$, seguidos de problemas emocionais (54,4\%), de relacionamento (52\%) e atenção/ hiperatividade $(45,6 \%)$. Diante disso, tornase urgente $o$ apoio psicossocial para $o$ acompanhamento da evolução dos sintomas, bem como para tentar amenizá-los nesses indivíduos. ${ }^{11,12}$

Foi relatada em estudo evidencia a fragilidade na articulação da rede no sistema de saúde representada por uma família composta por cinco pessoas (mãe, padrasto, irmão e duas irmãs), em que o padrasto agredia fisicamente a criança mais nova, que fugia de casa várias vezes, tentando sair de tal ambiente insalubre. ${ }^{6}$

\begin{abstract}
"Numa das ocasiões, a menina procurou abrigo na residência de um catador de papelão que ficava próximo à sua casa. Em troca, a criança o acompanhava diariamente no trabalho pelas ruas, sem acesso regular à alimentação, cuidados básicos de higiene e também sem frequentar a escola. Assim como nas ocasiões anteriores, quando soube onde se encontrava a filha, a mãe foi buscá-la, mas não obteve sucesso. Mesmo após ter acionado a polícia, a mãe sofreu ameaças do vizinho por insistir em levar a filha. Segundo relata, no momento em que ela tentou explicar a situação aos policiais, eles orientaram-na a retornar para casa, supondo que esse vizinho dizia a verdade. Sem argumentos, sentiu-se coagida, obedeceu aos policiais e voltou para casa sem a filha".
\end{abstract}

Nesse caso, a família, em vez de ser o alvo do cuidado das redes de atenção, foi considerada única responsável por seu aparente desajuste, visto que a abordagem da segurança pública foi extremamente ameaçadora. Assim, a família ficou na expectativa de uma ação mais assertiva da
Polícia e do Conselho Tutelar, o que não ocorreu, evidenciando, mais uma vez, a desarticulação entre os órgãos de apoio à criança e ao adolescente e suas famílias.

\section{Consequência dos maus-tratos a crianças e adolescentes}

Os maus-tratos contra crianças e adolescentes configuram um problema de saúde pública, tendo em vista sua recorrência e a gravidade dos abusos sofridos pelas vítimas. A exposição de crianças e adolescentes ao abuso físico, emocional e à negligência causam uma série de transtornos a esses jovens, os quais interferem definitivamente no seu futuro, como o aumento do risco de desenvolverem distúrbios sociais e psicológicos: álcool, drogas, depressão, ansiedade, transtorno do pânico. ${ }^{13}$

Também foi observado que abuso físico, emocional e negligência estão relacionados a um risco três vezes maior de desenvolver distúrbios alimentares nas vítimas infantojuvenis. Já o abuso físico, por si só, apresenta um risco cinco vezes maior de desenvolver bulimia nervosa, de acordo com os critérios diagnósticos do Manual Diagnóstico e Estatístico dos Transtornos Mentais (DSM). ${ }^{13}$

A incidência da violência infantil aumenta no País, mas a incompreensão, por parte dos parentes e também dos profissionais da Atenção Básica, da dimensão e dos efeitos dessa prática, tem um custo alto para as vítimas e para sociedade. ${ }^{13}$ Assim, intensifica-se a importância do ECA como assegurador dos direitos desse grupo, garantindo proteção contra quaisquer tipos de violência, seja física, psicológica ou sexual. 5,13 


\section{CONSIDERAÇÕES FINAIS}

Em linhas gerais, as leis de proteção e os estatutos como o ECA e os demais órgãos responsáveis (Conselho Tutelar, Ministério Público, Defensoria Pública, Justiça da Infância e Juventude e Delegacias Especializadas) existem para garantir a proteção à criança e ao adolescente vítimas de violência doméstica, oferecendo maneiras para que seus direitos sejam respeitados. Entretanto, na prática, notou-se uma desarticulação entre estes, sendo um obstáculo para a atenção integral às vítimas.

A percepção dos profissionais acerca dos mecanismos da violência doméstica é falha devido à má instrução, sendo importante uma formação mais abrangente no que tange à problemática.

Visto que a violência intrafamiliar contra crianças e adolescentes é de grande notoriedade e prevalência, é indispensável

\section{REFERÊNCIAS BIBLIOGRÁFICAS}

1. Melo RA, Roque EMST, Freitas LA, Carlos DM, Aragão AS, Ferriani MGC. Rede de proteção na assistência às crianças, adolescentes e suas famílias em situação de violência. Rev. Gaúcha Enferm. 2020; 41; e20190380.

2. Zambon MP, Jacintho ACA, Medeiros MM, Guglielminetti R, Marmo DB. Violência doméstica contra crianças e adolescentes: um desafio. Rev Assoc Med Bras. 2012; 58(4): 465-64.

3. World Vision. Covid-19 aftershocks: a perfect storm: millions more children at risk of violence under lockdown and into the 'new normal'. 2020.

4. Santos, P. P. G. A amizade infantil e a tirania do mundo dos adultos em "A ilha dos gatos que haja a promoção de medidas protetivas que não se restrinjam apenas a denúncias de maus tratos, mas que incluam métodos socioeducativos que visem a uma reconstrução da mentalidade social vigente, em que parentes recorrem à violência como método punitivista e educativo.

Logo, é fundamental a realização de mais pesquisas acerca do apoio psicossocial na Atenção Primária à Saúde como fonte de informação para subsidiar políticas, programas e estratégias de promoção da saúde e prevenção desse tipo de violência. Ademais, são necessárias ações intersetoriais, para além do setor saúde, que conduzam as vítimas ao cuidado integral, fornecendo apoio psicossocial e comunicação entre os sistemas de referência e contrarreferência e outros órgãos de proteção à criança e ao adolescente.

pingados". Muiraquitã: Rev. De Letras E Humanidades. 2020; 8 (2): 203-16.

5. Brasil. Lei n. 8.069, de 13 de julho de 1990. Dispõe sobre o Estatuto da Criança e do Adolescente e dá outras providências. Diário Oficial da União. 13 jul 1990.

6. Macedo EOS, Conceição MIG. Atendimento psicossocial a crianças e adolescentes em situação de violência: o psicólogo e a rede de atenção. Pesqui. Prát. Psicossociais. 2017; 12 (1): $129-46$.

7. Carvalho J, Duarte MLC, Glanzner, CH.Cuidado em saúde mental infantil no contexto da Estratégia da Saúde da Família: estudo avaliativo. Rev Gaúcha Enferm. 2020; 41(esp): e20190113. 
8. Condori PLP, Nascimento $S C L$, Mitie ABD, Pizzatto E, Mazza VA, Buffon MCM. Maus-tratos na infância e adolescência: percepção e conduta de profissionais de nível superior que atuam na Estratégia Saúde da Família. RSBO. 2018; 15(1): 34-40.

9. Shek G, Silva MRS, Lacharité C, Cézar-Vaz MR, Bueno MEN, Ventura J. Práticas profissionais que silenciam a violência intrafamiliar contra crianças e adolescentes. Texto Contexto Enferm. 2018, 27(1): e1680016.

10. Almeida AHV, Silva MLCA, Musse JO, Marques JAM. A responsabilidade dos profissionais de saúde na notificação dos casos de violência contra crianças e adolescentes de acordo com seus códigos de ética. ArqOdontol. 2012; 48(2): 102-15.
11. Hildebrand NA, Celeri EHRV, Morcillo AM, Zanolli ML. Violência doméstica e risco para problemas de saúde mental em crianças e adolescentes. Psicol Reflex Crit. 2015; 28(2): 21321.

12. Teixeira MR, Couto MCV, Delgado PGB. Atenção básica e cuidado colaborativo na atenção psicossocial de crianças e adolescentes: facilitadores e barreiras. Ciênc Saúde Colet. 2017; 22(6): 1933-42.

13. Norman RE, Byambaa M, De R, Butchart A, Scott J, Vos T. The long-term health consequences of child physical abuse, emotional abuse, and neglect: a systematic review and meta-analysis. PLoSMed. 2012; 9(11): e1001349. 